\title{
Perspectives on Deprescribing Communication in Primary Care
}

J Gen Intern Med 36(4):1122

DOI: $10.1007 / \mathrm{s} 11606-020-06377-\mathrm{x}$

(c) Society of General Internal Medicine 2021

$\mathrm{O}$ PTIMIZE $^{1}$ seeks to provide patients, care partners, and clinicians with tools - educational brochures and tip sheets-to foster thoughtful conversations about deprescribing. We agree that accurate medication reconciliation is an essential first step to such conversations. A pharmacy notebook such as the one you describe, involving indications for each medicine and patient memos with reasons for discontinuation, could be extremely useful. We would be very interested to see this approach tested as part of a deprescribing intervention, as it is consistent with engaging patients and caregivers and increasing trusting conversations.

Ariel R. Green, $M D, M P H, P h D^{1}$

Cynthia M. Boyd, $M D, M P H^{1}$

Kathy S. Gleason, $P h D^{2}$

Leslie Wright, $M A^{2}$

Courtney R. Kraus, $\mathrm{MSPH}^{2}$

Ruth Bedoy, $B S^{2}$

Bianca Sanchez, $\mathrm{BS}^{3}$

Jonathan Norton, $B S^{1}$

Orla C. Sheehan, $\mathrm{MD}, \mathrm{Ph} \mathrm{D}^{1}$

Jennifer L. Wolff, $\mathrm{Ph} \mathrm{D}^{4}$

Emily Reeve, BPharm(Hons) $\mathrm{PhD}^{5}$

Matthew L. Maciejewski, $P h D^{6,7}$

Linda A. Weffald, PharmD ${ }^{8}$

Elizabeth A. Bayliss, MD, MSPH ${ }^{2,9}$

${ }^{1}$ Division of Geriatric Medicine and Gerontology,

Department of Medicine, Johns Hopkins

University School of Medicine,

Baltimore, MD, USA
${ }^{2}$ Institute for Health Research, Kaiser Permanente Colorado,

Aurora, CO, USA

${ }^{3}$ University of Colorado School of Medicine,

Aurora, CO, USA

${ }^{4}$ Roger C. Lipitz Center for Integrated Health Care, Department of Health Policy and Management, Johns Hopkins Bloomberg School of Public Health,

Baltimore, MD, USA

${ }^{5}$ Quality Use of Medicines and Pharmacy Research Centre, UniSA: Clinical and Health Sciences, University of South Australia,

, SAAdelaide, Australia

${ }^{6}$ Durham Center of Innovation to Accelerate Discovery and Practice Transformation (ADAPT), Durham Veterans Affairs Medical Center,

Durham, NC, USA

${ }^{7}$ Department of Population Health Sciences, Duke University Medical Center,

Durham, NC, USA

${ }^{8}$ Department of Clinical Pharmacy, Kaiser Permanente Colorado,

Aurora, CO, USA

${ }^{9}$ Department of Family Medicine, University of Colorado School of Medicine,

Aurora, CO, USA

Corresponding Author: Ariel R. Green, MD, MPH, PhD; Division of Geriatric Medicine and Gerontology, Department of Medicine, Johns Hopkins University School of Medicine, Baltimore, MD, USA (e-mail: ariel@jhmi.edu).

\section{REFERENCE}

1. Bayliss EA, Shetterly SM, Drace ML, et al. The OPTIMIZE patient- and family-centered, primary care-based deprescribing intervention for older adults with dementia or mild cognitive impairment and multiple chronic conditions: study protocol for a pragmatic cluster randomized controlled trial. Trials. 2020;21(1):542.

Publisher's Note: Springer Nature remains neutral with regard to jurisdictional claims in published maps and institutional affiliations. 